\title{
Red palm weevil monitoring and early warning system
}

\author{
Keith Cressman \\ FAO, Rome, Italy, email: keith.cressman@fao.org
}

\begin{abstract}
Cressman, K. 2019. Red palm weevil monitoring and early warning system. Arab Journal of Plant Protection, 37(2): 203-204.

To improve effective and sound decisions related to red palm weevil management, FAO has developed an initial beta version of a global RPW monitoring and early warning system to help farmers and national authorities respond to this important transboundary pest on date, coconut and ornamental palms in Africa, Asia and Europe. The system consists of the SusaHamra mobile application for data collection in the field and a GIS-based online platform for data analysis and mapping.

Keywords: RPW, monitoring, forecasting, warning system.
\end{abstract}

\section{Introduction}

There are currently very few tools available that can be used to systematically collect standard geo-referenced data in the field for the regular monitoring and management of the Red Palm Weevil (RPW) (Cressman and Viprathi, 2017; FAO 2017a). In the absence of such data and analysis, it is nearly impossible to take effective and technically sound decisions (Fajardo et al., 2017; FAO2017b). To address this critical shortcoming, FAO has developed an initial beta version of a global RPW monitoring and early warning system to help farmers and national authorities respond to this important transboundary pest on date, coconut and ornamental palms in Africa, Asia and Europe. The system consists of the SusaHamra mobile app for data collection in the field and a GIS-based online platform for data analysis and mapping. Based on lessons learned from FAO's long experience in developing and operating global monitoring and early warning systems for Desert Locust and more recently for the Fall Armyworm, the mobile app for smartphones has been developed as a tool that can be used every time palms are inspected or treated, or when pheromone traps are checked for RPW. The collected data feed into a centralised database for mapping and analytics that show the current situation and its geo-temporal spread. The data will be valuable to decision-makers and researchers within countries and throughout the world. In addition, FAO is developing a protocol for mapping palm farms based on remote sensing and machine-learning, artificial intelligence that will be integrated into the system to supplement analysis of the field data. SusaHamra will also include educational material on RPW and its management, and be available in English, Arabic and other local languages. The RPW monitoring and early warning system has been designed in a dynamic manner in order to account for the changing needs of users and countries over time. FAO offers its unique role in developing and maintaining a global tool that can provide impartial and unbiased advice to countries or farmers in a sustainable manner.

\section{SusaHamra mobile application}

The beta version of the app consists of three sections: information, palms and traps. The information section contains the name of the field, the date of the inspection and the type of inspection (palms or traps). The palms section contains data on the presence/absence of RPW, visible symptoms, tissue damage, inspection method, control undertaken and by whom. The traps section contains data on catches and trap servicing (by whom, last date, lure replacement).

Users must first register before they can use the app. The one-time only registration process consists of entering basic information about the farm, palms and traps. New palms and traps can be entered at any time. This will allow easy inspections of individual palms and trap in order to monitor the distribution and spread of RPWM in farms. The app is initially available in English and Arabic. It is free and will be eventually available for download from the Google Play Store and the Apple App Store.

There are also specific user modes for data validation module to maintain a high level of data quality and for additional language translations.

Substantial field testing, validation and refinement are to be done before SusaHamra is ready for release and operational use. The first field evaluation was carried out in March 2019 in Oman. This will be further refined and additional feedback will be obtained from users in other countries before finalizing the app.

SusaHamra should be used every time palms and traps are inspected for RPW.

\section{Global platform}

The global platform consists of a centralised database in the cloud and maps and graphical analytics for the presentation and analysis of the data collected by SusaHamra. The platform is managed by FAO Headquarters in Rome and utilises PowerBI software. The geo-referenced data are presented as maps, graphs and data tables. The maps can be 
zoomed from the global to country, province and farm level. The corresponding analytical graphs will be displayed. If a specific country, province or farm is selected, the graphs and data tables will change accordingly. This is an easy way not only to display data but to see data relationships such as RPW presence and inspection method or visible symptoms, or RPW catches by lure or trap type. An example of the platform is the Fall Armyworm Monitoring and Early Warning System (FAMEWS) that was developed and maintained by FAO (www.fao.org/fall-armyworm).

\section{Palm mapping}

FAO is using a machine-learning, artificial intelligence approach to map individual palm trees based on Google Tensor Flow and lessons learned from the FAMEWS Nuru app (www.fao.org/fall-arymworm/) by Penn State University. This approach relies on thousands of palm photos that are used for learning by the computer. This knowledge is then applied to high-resolution geo-referenced satellite imagery and digital photography to map the palms. The initial work focuses on two sample areas, one that consists homogenous of palm farms (Gassim, Saudi Arabia) and one that is more urban and heterogenous (Al Ahsa, Saudi Arabia). The results will be upscaled and applied to larger areas in order to provide a base map of individual palms that can be used in SusaHamra, the global platform and geographic information systems.

\section{Conclusion}

The development of the RPW monitoring and early warning system is funded by FAO seed money, supplemented by contributions to the Global RPW Trust Fund. The system is flexible and adaptable to meet users' constantly changing needs. The SusaHamra mobile app and global platform, are made available to the global community for free as tools to more effectively monitor and manage RPW. There are no registration, monthly subscription, data storage or product fees, which sets apart this system from commercial products and services. In addition, hosting the data and global platform at FAO ensures sustainability and free access within a secure environment that does not compromise individual privacy. FAO remains a trusted, non-profit partner with global reach. It is objective, impartial, unbiased, apolitical and neutral. It uses cutting-edge and the latest innovative technologies to develop free tools, systems and platforms that can quickly and easily be upscaled. FAO has more than 75 years of Desert Locust monitoring and early warning experience that can also be applied to similar systems for RPW.

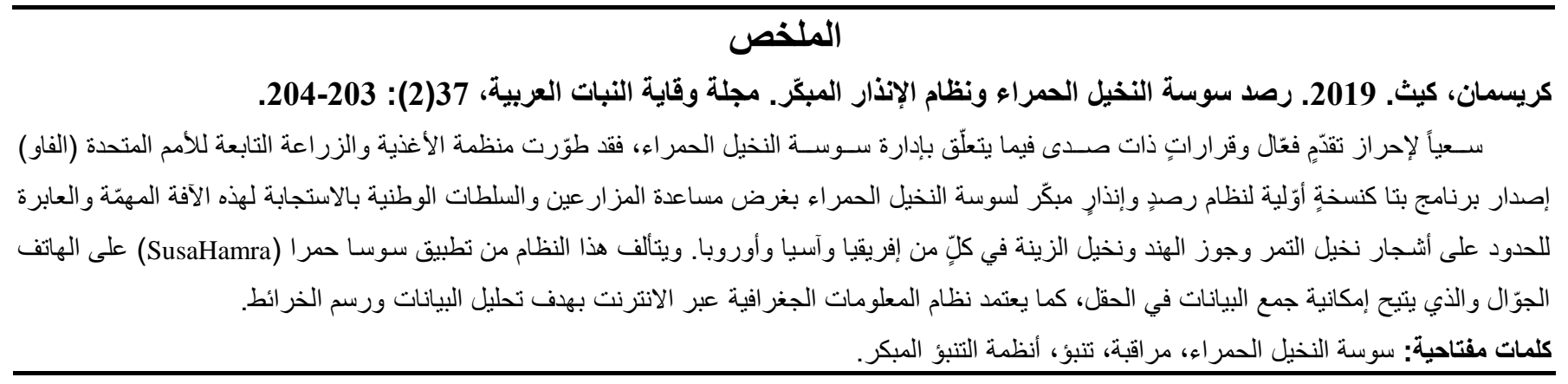

\section{References}

Cressman, K. and K. Viparthi. 2017. Innovative solutions using modern technologies for better management, control and analysis of Rhynchophorus ferrugineus eradication. Presented at the "Scientific Consultation and High-Level Meeting on Red Palm Weevil Management", organized by FAO and CIHEAM, 2931 March, 2017, Rome, Italy.

Fajardo, M., J.A. Guerra, L. Barroso, M. Morales and R. Martín. 2017. Use of GIS (Geographical Information System) for data management and analysis in a Rhynchophorus ferrugineus eradication program. Presented at the "Scientific Consultation and High-
Level Meeting on Red Palm Weevil Management”, organized by FAO and CIHEAM, 29-31 March, 2017, Rome, Italy.

FAO. 2017a. Current Situation of Red Palm Weevil in the NENA Region [Current situation of management practices, challenges/weaknesses and available research and technologies for its improvement]. http://www.fao.org/3/a-ms664e.pdf (accessed on 25 March, 2019).

FAO. 2017b. Framework Strategy for Eradication of Red Palm Weevil. http://www.fao.org/3/a-mt054e.pdf (accessed on 21 February, 2019). 